\title{
Ultrafast dynamic imaging of thermal and acoustic dyn- amics in nanosystems using a tabletop high harmonic source
}

\author{
Charles Bevis ${ }^{1}$, Robert Karl Jr. ${ }^{1}$, Giulia F. Mancini ${ }^{1,2}$, Dennis Gardner ${ }^{1}$, Elisabeth Shanblatt ${ }^{1}$, Joshua \\ Knobloch $^{1}$, Travis Frazer ${ }^{1}$, Jorge N. Hernandez-Charpak ${ }^{1}$, Begoña Abad Mayor ${ }^{1}$, Michael Tanksalvala ${ }^{1}$, \\ Christina Porter $^{1}$, Daniel Adams ${ }^{1}$, Henry Kapteyn ${ }^{1}$ and Margaret M. Murnane ${ }^{1}$ \\ ${ }_{2}^{1}$ JILA, 440 UCB, University of Colorado, Boulder, CO 80309 USA \\ 2 Paul Scherrer Institut, WSLA/210, 5232 PSI Villigen, Switzerland.
}

\begin{abstract}
We demonstrate the first stroboscopic full-field EUV nanoscope using high harmonics. We image the propagation of thermal and surface acoustic waves in nickel with $80 \mathrm{~nm}$ transverse, $0.5 \AA$ axial, and $10 \mathrm{fs}$ resolution.
\end{abstract}

\section{Introduction}

With the rapid downscaling of nano-devices and nano-enhanced materials, nanofabrication has now outpaced the capabilities of most conventional metrology techniques. However, at dimensions $<100 \mathrm{~nm}$, smart design of functional nanosystems is challenging because bulk macroscopic models can no longer accurately predict heat, charge or spin transport, or the mechanical properties of doped or nano-structured materials [1]. Moreover, many nano-structure materials are opaque to visible light. As a result, there is a critical need for new functional microscopes that can stroboscopically image with high spatial and temporal resolution, through opaque overlayers, with elemental, chemical and magnetic contrast.

In this work, we present the first dynamic, full-field imaging system using tabletop extreme ultraviolet (EUV) high harmonic (HHG) beams. With this instrument, we directly visualize nanoscale thermal and acoustic wave propagation with $80 \mathrm{~nm}$ spatial resolution, $0.5 \AA$ axial resolution, and $10 \mathrm{fs}$ temporal resolution. To achieve this, we combine ptychography coherent diffractive imaging (CDI) [2,3] with a tabletop HHG source. After exciting the nanosystem with a 23 fs laser pulse, we stroboscopically image the resulting thermal expansion and heat flow, as well as the impulsive longitudinal and transverse acoustic wave propagation of nickel nanostructures patterned on a silicon substrate. This work is a first step toward combining the ultra-high spatial resolution possible using EUV-CDI with the ultra-high femtosecond temporal resolution of HHG sources [4,5]. This advance can uncover new basic understanding of nanoscale materials, while also providing new tools for functional imaging to inform next-generation device design at dimensions $<<100 \mathrm{~nm}$.

\section{Experimental Methods}

Our experimental setup is shown in Fig. 1(a). To generate the excitation and pump pulse for our microscope, we used the output of a Ti:Sapphire oscillator which was subsequently amplified to a pulse energy of $1.5 \mathrm{~mJ}$, repetition rate of $5 \mathrm{kHZ}$, pulse width of $23 \mathrm{fs}$, and central- $\lambda$ of $785 \mathrm{~nm}$. The resulting beam was split with one arm directed into an argon filled fiber where the light was upconverted via the HHG process. The residual red light was absorbed with two super-polished Si mirrors set at Brewster's angle combined with an aluminium filter. The resulting HHG beam is directed into the imaging chamber where our CDI microscope resides. Inside the chamber, a single harmonic of $\lambda=28.9 \mathrm{~nm}$ is selected with two multiplayer mirrors. An ellipsoidal mirror focuses the harmonic light on to the sample with a spot size of $21 \mu \mathrm{m}$ x $21 \mu \mathrm{m}$. Simultaneously, the sample is pumped at the fundamental wavelength of $785 \mathrm{~nm}$ with second arm of the split beam, which has been directed through a delay stage. The resulting diffracted light is collected by a CCD detector place $36.5 \mathrm{~mm}$ from the sample surface. An additional $195 \mathrm{~nm}$ thick aluminum filter was placed in front of the camera to filter any IR light reflected by the sample towards the detector. The chamber geometry is shown in Fig. 2(b).

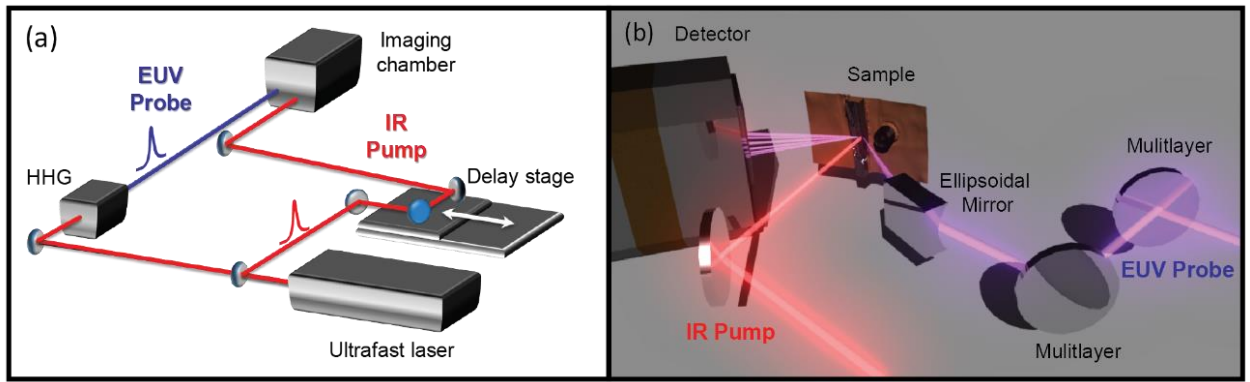

Fig. 1. The experimental setup. (a) The beam geometry of the two experimental arms. (b) A view inside the imaging chamber, showing the CDI microscope configured in a pump probe geometry.

\footnotetext{
*Corresponding author:charles.bevis@colorado.edu
} 
The sample we investigated for this work consists of $20 \mathrm{~nm}$ tall nickel nanostructures on a silicon substrate. The structure investigated was a triangle with a width of $5 \mu \mathrm{m}$, length of $53 \mu \mathrm{m}$ and a grating with a pitch of $3 \mu \mathrm{m}$. To excite the thermal dynamics, the sample was uniformly illuminated with the IR pump at a fluence of $8.4 \mathrm{~mJ} / \mathrm{cm}^{2}$ with a $500 \mu \mathrm{m}$ spot size. Diffraction from the sample was collected at different time delays. During each time delay, the sample was imaged using ptychography CDI, where multiple diffraction patterns are recorded from overlapping fields-of-view of the sample. The dynamic response of the sample is determined by examining the changes in the diffraction pattern at different delay times between the pump and probe pulses. The transient response displays a sharp rise at zero delay time corresponding to a $<1$ ps thermal expansion, followed by oscillations due to surface acoustic waves launched within the silicon substrate and nickel features.

\section{Analysis and Results}

The dynamic diffraction efficiency of the nickel lines was process analogous to the process outlined in [1], by comparing the normalized difference in the power contained in the $1^{\text {st }}$ order diffraction peak compared to the $0^{\text {th }}$ order. We validated this measurement, by extracting the same signal from a single diffraction pattern taken during a ptychography scan. We see good agreement between these two measurements, likely indicating that we are indeed imaging the acoustic dynamics we would expect to see through a stroboscopic measurement.

When the spatial shape of the feature becomes less uniform, we begin to expect different dynamics at different spatial locations. This information cannot be extract using the previous method because we are spatially averaged. In order to observe these dynamics, we use ptychography CDI to reconstruct both the amplitude and phase of the nickel triangle at many different time delays. With prior knowledge of the sample composition, the reconstructed phase gives us information about the height at each delay time. Assembling these reconstructions allows us to observe the initial thermal expansion and subsequent surface acoustic waves in both the nickel feature and the silicon substrate, as shown in Fig. 2. We note that we are able to observe complex acoustic wave propagation due to the non-uniform width (V shape) of the sample. A full 3D simulation of these dynamics would require enormous computation power, and thus we must approximate in the simulation by viewing the dynamics of a single transverse slice through the triangle. Figure 2(e) plots the result of our simulation, which is in good agreement with our data.
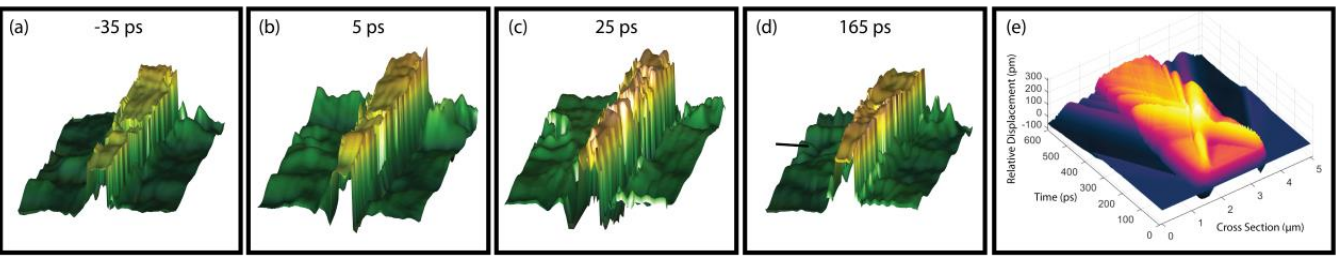

Fig. 2. (a-d) Ptychography CDI reconstructions of a subsection of the Ni triangle at different time delays. The different panel display the spatially resolved acoustic and thermal dynamics of this sample. The scale bar in (d) is $2 \mu \mathrm{m}$ in the lateral direction and $0.5 \mathrm{~nm}$ in the vertical. The scale is the same in each image (a-d). (e) A simulation displaying the acoustic dynamics through a transverse slice of the triangle.

\section{Conclusion}

We have demonstrated the first full-field imaging system capable of capturing the dynamic properties of nanoscale systems with nm spatial and fs temporal resolution. Higher spatial resolution imaging in the transverse direction can be readily achieved in future work, as we have already demonstrated $0.9 \lambda(12.6 \mathrm{~nm})$ spatial resolution using shorter wavelength illumination beams. With this result, we take the first step toward directly visualizing the complex nature of nanoscale charge, spin, and heat transport with nm spatial and fs temporal resolution.

\section{Acknowledgments}

The authors gratefully acknowledge support from DARPA PULSE W31P4Q-13-1-0015, a Gordon and Betty Moore Foundation EPiQS Initiative through Grant GBMF4538, an NSF STROBE DMR- 1548924, an NSF GRFP, an NDSEG, and an SRC fellowship.

\section{References}

[1] K. Hoogeboom-Pot, J. Hernandez-Charpak, X. Gu, T. Frazer, E. Anderson, W. Chao, R. Falcone, R. Yang, M. Murnane, H. Kapteyn, and D. Nardi, "A new regime of nanoscale thermal transport: Collective diffusion increases dissipation efficiency," PNAS 112, 4846(2015).

[2] P. Thibault., M Dierolf, O Bunk, A Menzel, and F Pfeiffer, "Probe retrieval in ptychographic coherent diffractive imaging," Ultramicroscopy, 109, 338 (2009).

[3] M. Maiden and J. Rodenburg, “An improved ptychographical phase retrieval algorithm for diffractive imaging," Ultramicroscopy 109 1256 (2009).

[4] C. Porter, M. Tanksalvala, M. Gerrity, G. Miley, X. Zhang, C. Bevis, E. Shanblatt, R. Karl, M. Murnane, D. Adams, and H. Kapteyn, "General-purpose, wide field-of-view reflection imaging with a tabletop $13 \mathrm{~nm}$ light source," Optica, 4, 1552 (2017).

[5] D. Gardner, M. Tanksalvala, E. Shanblatt, X. Zhang, B. Galloway, C. Porter, R. Karl, C. Bevis, D. Adams, H. Kapteyn, M. Murnane, and G. Mancini, "Subwavelength coherent imaging of periodic samples using a $13.5 \mathrm{~nm}$ tabletop high-harmonic light source," Nat. Photonics, 11, 259 (2017). 